\title{
Ergotherapie
}

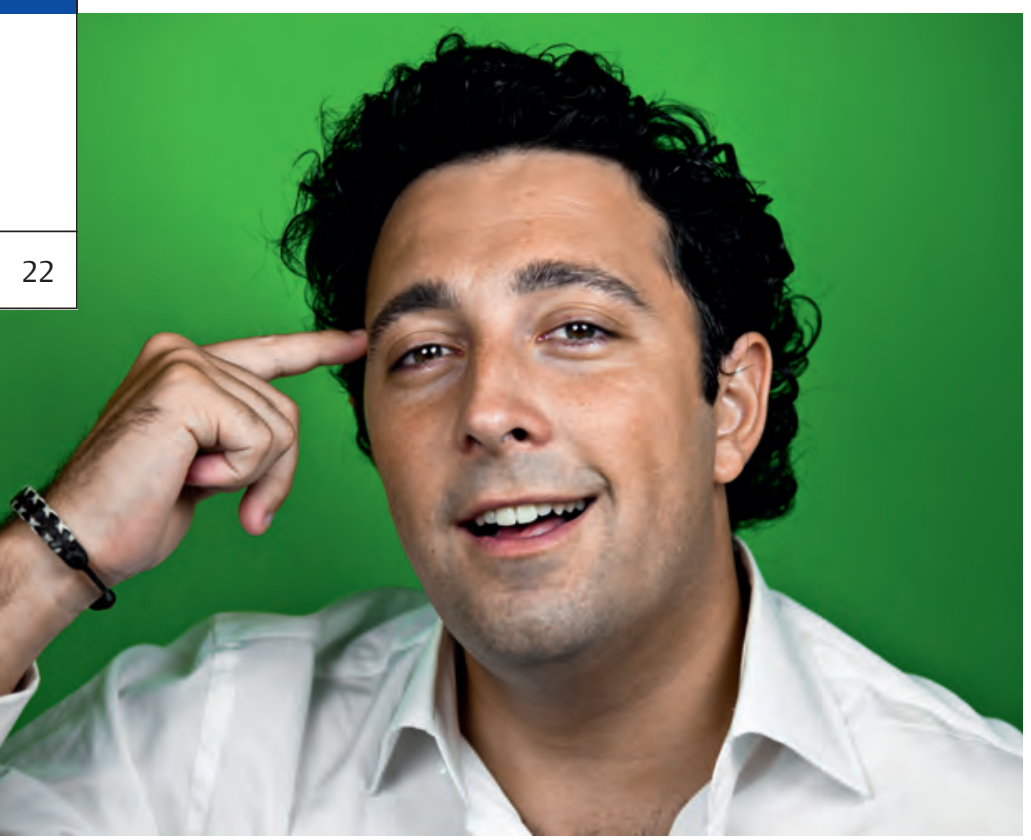

D amit Patienten mit einer Apraxie (॰ „Definition“) frühzeitig eine adäquate Therapie erhalten, muss die Erkrankung standardisiert und zuverlässig erfasst werden [1, 4]. Zusammen mit meiner Forschungsgruppe entwickelte ich 2011 den Apraxia Screen of TULIA (AST), mit dem Therapeuten schnell herausfinden können, ob ein Patient unter einer Apraxie leidet [5, 6]. Mittlerweile hat sich das validierte Screening-Instrument in Kliniken und in Rehazentren durchgesetzt.

In drei Minuten zum Ergebnis > Der AST basiert auf einem umfangreichen Test mit 48 Items namens TULIA (Test of Upper Limb Apraxia), den meine Kollegen und ich 2010 entwickelten [3]. Für den AST reduzierten wir die Anzahl der Items auf zwölf. Damit beinhaltet das Assessment nun drei kommunikative, acht werkzeugbezogene Gesten und eine bedeutungslose Geste ( $\odot$ „Apraxia Screen of TULIA (AST)“). Im Vergleich zum TULIA geht der AST viel schneller und ist praxistauglicher. Außerdem haben wir aus den 48 Items die zwölf ausgewählt, die in Bezug auf die Sensitivität und Spezifität am besten geeignet sind, um eine Apraxie festzustellen.

Die Items werden dichotom bewertet (richtig = ein Punkt oder falsch = kein Punkt), somit kann das Endresultat zwischen 0 und 12

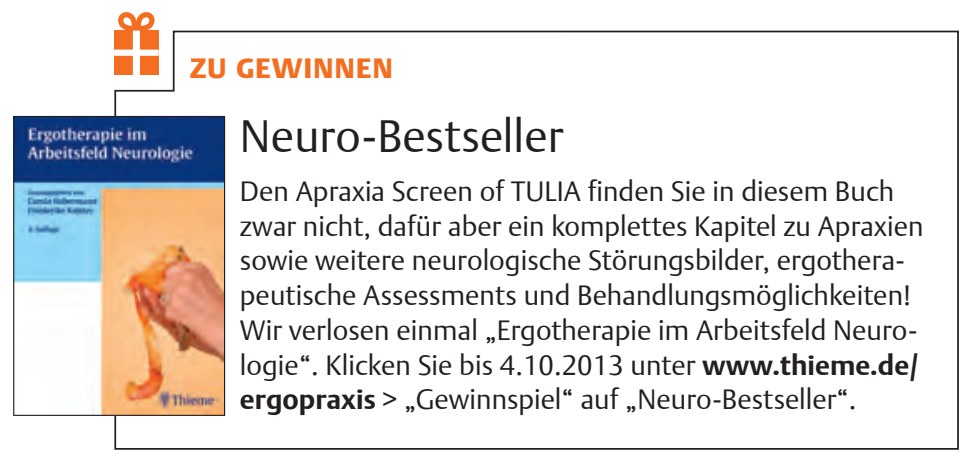

\section{Gesten testen}

ASSESSMENT: APRAXIA SCREEN OF TULIA Das

brandneue Assessment „Apraxia Screen of TULIA“

geht schnell und bringt zuverlässige Ergebnisse. Anhand von 12 Handbewegungen - darunter auch „Zeigen Sie, dass jemand spinnt“ - können Ergotherapeuten herausfinden, ob ein Patient unter einer Apraxie leidet.

Punkten liegen. Ein Wert $<9$ deutet auf eine leichte Apraxie hin, ein Ergebnis $<5$ Punkten auf eine schwere Apraxie.

Der Test dauert maximal drei Minuten. Der Patient sitzt an der Bettkante, im Stuhl oder Rollstuhl der Ergotherapeutin gegenüber. Bei den Imitationsaufgaben macht die Therapeutin die Gesten vor. Anschließend soll der Patient die Geste spiegelbildlich nachahmen. Beim pantomimischen Teil erklärt die Untersucherin die Aufgabe verbal, danach führt der Patient sie aus.

Bei Patienten mit Parkinson-Erkrankungen oder kortikobasalen Degenerationen sowie bei Patienten nach Schlaganfall, die keine Parese haben, wird die Apraxie immer bilateral getestet. Zwar wirkt sie sich in der Regel auf beide Gliedmaßen gleichermaßen aus, doch manche Patienten zeigen auch eine asymmetrische Apraxie. Daher empfiehlt es sich, den AST stets bilateral zu testen. Bei Patienten mit einer Hemiparese wird nur die ipsilaterale, nichtparetische Seite untersucht, da auf der kontralateralen Seite die apraktischen Defizite durch Schwäche und Sensibilitätsverlust maskiert werden können. Patienten mit einer schweren Aphasie sind möglicherweise nicht in der Lage, die Pantomimeaufgaben auszuführen. In dem Fall werden nur die sieben Imitationsübungen ausgewertet: Eine Apraxie liegt hier bei einem Wert < 5 vor.

Zuverlässig und valide > Die interne Konsistenz des AST ist sehr hoch [5]. Das weist darauf hin, dass alle zwölf Items des Screenings ein ähnliches Konstrukt, nämlich die Apraxie, messen. Bei einer wiederholten Erhebung des AST bei Menschen mit Schlaganfall zeigte sich eine gute Test-Retest-Reliabilität [5]. Zudem ist die diagnostische Genauigkeit des Assessments sehr hoch: Der AST hat eine Spezifität von 100 Prozent und eine Sensitivität von 95 Prozent.

Kritisch anzumerken ist, dass der AST keine Aufgaben zum tatsächlichen Objektgebrauch beinhaltet. Daher kann es sein, dass eine selektive Störung des reellen Objektgebrauchs unentdeckt bleibt. Eine selektive Beeinträchtigung ist zwar bei Patienten mit 


\section{APRAXIA SCREEN OF TULIA (AST)}

\section{Die zwölf Items}

> Imitation: Allgemeine Instruktion: „Sieben Gesten werden spiegelbildlich vorgemacht, ahmen Sie diese so präzise wie möglich nach.“

\begin{tabular}{|c|c|c|}
\hline & rechts & links \\
\hline $\begin{array}{l}\text { 1. Daumenkuppe gestreckt auf die Stirn, Finger } \\
\text { zeigen nach oben }\end{array}$ & & \\
\hline 2. Staub von der Schulter wischen & & \\
\hline
\end{tabular}

Zusätzliche Instruktion: „Für die nächsten fünf Bewegungen stellen Sie sich vor, einen Gegenstand in der Hand zu halten; gebrauchen Sie nicht Ihre Finger als Werkzeug."

\begin{tabular}{l|l|l|}
\hline 3. Aus einem Schnapsglas trinken & & \\
\hline 4. Eine Zigarette rauchen & & \\
\hline 5. Einen Hammer benutzen & & \\
\hline 6. Eine Schere benutzen & & \\
\hline 7. Einen Poststempel benutzen & & \\
\hline
\end{tabular}

> Pantomime: Allgemeine Instruktion: „Jetzt werden Ihnen Gesten laut vorgesagt. Hören Sie gut zu und führen Sie die Gesten so präzise wie möglich aus.“

\begin{tabular}{l|l|l|}
\hline 8 . „Zeigen Sie, dass jemand spinnt.“ $*$ & & \\
\hline 9. „Drohen Sie mit der Hand.“ ${ }^{* *}$ & & \\
\hline
\end{tabular}

Zusätzliche Instruktion: „Jetzt stellen Sie sich wieder vor, einen Gegenstand in der Hand zu halten; gebrauchen Sie nicht Ihre Finger."

\begin{tabular}{l|l|l}
\hline $10 .$, „Putzen Sie sich die Zähne.“ & & \\
\hline $11 .$, Kämmen Sie Ihre Haare.“ & & \\
\hline 12. „Benutzen Sie einen Schraubenzieher.“ & & \\
\hline Gesamtscore & & \\
\hline * repetitives Klopfen des Zeigefingers an der Schläfe (auch eine drehende Bewegung \\
des Zeigefingers ist korrekt)
\end{tabular}

$\Rightarrow$ Das Formular zum Assessment steht zum Download unter www.thieme-connect.de/ejournals/ergopraxis > „Ausgabe 9/13“.

Apraxie selten, dennoch ist es zu empfehlen, zusätzlich den reellen Objektgebrauch zu prüfen. Dies kann die Ergotherapeutin durch Beobachten des Patienten im klinischen Alltag herausfinden.

Leicht in den klinischen Alltag zu integrieren > Der AST ist ein valides Assessment, mit dem Ergo- und Physiotherapeuten zuverlässig, schnell und ohne Hilfsmittel eine Apraxie diagnostizieren können - sogar direkt am Bett des Patienten. Daher ist er leicht in den klinischen Alltag zu integrieren. Um einen Überblick darüber zu bekommen, wie sich die Apraxie auf den Alltag des Patienten auswirkt, empfiehlt es sich, ihn bei verschiedenen Aktivitäten des täglichen Lebens zu beobachten. Durch den AST ist eine objektive Erfassung der Störung garantiert, sodass apraxiespezifische Interventionen frühzeitig in die Wege geleitet werden können [1].

Tim Vanbellingen

\section{$\Rightarrow$ Das Literaturverzeichnis steht unter www.thieme-connect.de/ ejournals/ergopraxis > „Ausgabe 9/13“.}

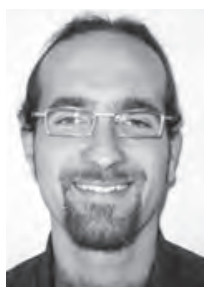

Dr. Tim Vanbellingen, PhD, ist Physiotherapeut und hat sein Studium in Belgien abgeschlossen. In seiner Masterarbeit schrieb er über die Entwicklung eines neuen Apraxie-Tests. Ende April 2013 hat er seine Doktorarbeit an der Universität Bern (Schweiz) abgeschlossen. Für diesen Artikel dankt er Noëmi Eggenberger für die Durchsicht des Manuskriptes.

\section{DEFINITION}

\section{Was ist eine Apraxie?}

Eine Apraxie ist eine kognitive motorische Störung, die folgende Handlungen beeinträchtigen kann [2]:

$>$ die Imitation von abstrakten, nichtsymbolischen Gesten

$>$ das pantomimische Darstellen eines Gegenstandsgebrauchs, zum Beispiel der Gebrauch eines Werkzeugs

$>$ der reelle Gebrauch von Werkzeugen und Objekten

Eine Apraxie ist nicht durch ein primär sensorisches oder motorisches Defizit ausgelöst und kann auch nicht durch eine Kommunikationsstörung, fehlende Motivation oder ein Aufmerksamkeitsdefizit erklärt werden [4]. Die Patienten haben erhebliche Schwierigkeiten bei verschiedenen Aktivitäten des täglichen Lebens (ADL), beispielsweise beim Ankleiden oder beim Schuhebinden.

Eine Apraxie wirkt sich negativ auf den Rehabilitationserfolg aus: So kehren beispielsweise Patienten nach Schlaganfall, die eine Apraxie bekommen, signifikant seltener in den Berufsalltag zurück als Patienten ohne dieses Störungsbild.

Die Prävalenz ist unter Patienten nach Schlaganfall besonders hoch: Rund 50 Prozent der Patienten, die einen linkshemisphärischen Insult erlitten haben, bekommen eine Apraxie. Des Weiteren kommt eine Apraxie gehäuft bei Patienten mit neurodegenerativen Störungen vor, zum Beispiel bei kortikobasalen Degenerationen [6, 7]. 